\title{
Comparison of the Accuracy of Age Estimation Methods on Saudi Arabian Population - A Cross Sectional Study
}

\author{
Sajna Ashraf1, Vinod Babu Mathew², Farah Masaad³, Shaheen Vilayil Shamsuddin, \\ Jimly James Kunjappu ${ }^{5}$, Ahmed Noor Alotaibi ${ }^{6}$
}

\begin{abstract}
${ }^{1}$ Department of Oral Medicine and Diagnostic Science, Al-Farabi Colleges, Riyadh, Saudi Arabia. ${ }^{2}$ Department of Restorative Dental Sciences, College of Dentistry, King Khalid University, Abha, Saudi, Arabia. ${ }^{3}$ Department of Oral Medicine and Diagnostic Science, Al-Farabi Colleges, Riyadh, Saudi Arabia. ${ }^{4}$ Department of Paediatric Dentistry and Orthodontic Sciences, College of Dentistry, King Khalid University, Abha, Saudi Arabia. ${ }^{5}$ Department of Periodontics and Community Dental Sciences, College of Dentistry, King Khalid University, Abha, Saudi Arabia. ${ }^{6}$ Smile World Dental Center, Riyadh, Saudi Arabia.
\end{abstract}

\section{ABSTRACT}

\section{BACKGROUND}

Identity and legal culpability hinges on age estimation in modern society. There are several biometric methods for accurate age determination that have validity in medico-legal proceedings globally. The accuracy of conventional methods should be reassessed periodically to ascertain validity in differing global societies. This study aims to evaluate the comparative accuracy of three age determination methods based on non-invasive radiographic odontometric data when applied to a sample of Saudi population.

\section{METHODS}

This cross-sectional study is done on 350 subjects of Saudi Arabian population, aged between 4 and 16 years. The same set of radiographs is used in all three methods and the dental age estimation is done by trained examiners who determine the age using Demirjian's, Willem's and the London Atlas Method. Statistical analysis is done using the IBM SPSS (Statistical Package for Social Science) software (version 19). Significance of differences between the chronological age and the estimated dental age was calculated using paired ' $\mathrm{t}$ ' test $(\mathrm{P}$ value of $<0.05$ was considered significant.

\section{RESULTS}

The chronological age was above estimated age by all three methods used to assess male samples. Significant difference was seen with William's method $(p=0.000)$ and the the London Atlas Method ( $\mathrm{p}=0.000)$, and no significant difference was seen with Demirjian's method $(p=0.102)$. Demirjian's method estimated the age above chronological age for females while the other two methods were below in their estimates. There were significant differences only between the London Atlas estimates and chronological age in females $(p=0.020)$.

\section{CONCLUSIONS}

Age estimated by Demirjian's method on Saudi population is most accurate among the methods tested. Boys showed more variations than girls when the ages were measured. Changing diet and socioeconomic factors influence growth and development of children, and this can alter the anticipated outcomes of age determination by mathematical methods.

\section{KEY WORDS}

Age Estimation, Saudi Arabian Population, Demirjian's Method, Willem's Method, London Atlas Method
Corresponding Author:

Dr. Sajna Ashraf,

Department of Oral Medicine and

Diagnositc Science, Al-Farabi Colleges,

Riyadh, Saudi Arabia.

E-mail: drsajnaashraf@gmail.com

DOI: $10.14260 /$ jemds/2020/590

How to Cite This Article:

Ashraf S, Mathew VB, Masaad F, et al. Comparison of the accuracy of age estimation methods on Saudi Arabian population - a cross sectional study. J Evolution Med Dent Sci 2020;9(37):27172721, DOI: $10.14260 /$ jemds/2020/590

Submission 20-04-2020,

Peer Review 07-08-2020,

Acceptance 12-08-2020,

Published 14-09-2020.

Copyright (C) 2020 JEMDS. This is an open access article distributed under Creative Commons Attribution License [Attribution 4.0 International (CC BY 4.0)] 


\section{BACKGROUND}

Identifying the age of an individual is an important procedure in forensic sciences. ${ }^{(1)}$ The need for positively determining the age of an individual has many uses in medical and legal fields. The positive age estimation of unknown individual's postmortem is different from ante-mortem analysis. Changes that occur in dental tissue remain for a far greater period than other human tissue due to its virtual indestructability. The histopathologic determination of the age changes in teeth and estimation of age is quite accurate but only applicable to postmortem analysis or extracted teeth form living individuals. This decreases its value for use in medical specialties, which deal with the living.(2,3) This brings into focus the importance of imaging tools for non-invasive estimation of age.

More reliable age estimation results are obtained from dental parameters when compared to the skeletal development estimation methods. This is especially applicable in children and adolescents, where the development is still ongoing.(3) Estimation of age in the pre-adult stage is of vital significance in determining the culpable age in legal proceedings. Thus, the need for reliable methods that corroborate the assumed chronological age is essential.

Literature reviews confirms that similar systematic approaches are followed to estimate the dental age maturation. The primary approach in dental age estimation has been to evaluate the stages of development of tooth follicles and to use the chronological developmental timetable during the mixed dentition phase. These parameters which have been studied clinically and radiographically in several studies have been matched to the known chronological age to form correlations that can be expressed as mathematical correction to derive one or the other. $(2,4)$ Based on dental maturation in children, age estimation techniques are divided into two: those using scoring systems and those using the atlas approach. ${ }^{(5)}$

The earlier methods focused on deriving a uniform applicable scale to estimate age depending on the score estimated from the stages of development that were visualized radiographically. These were developed on populations that were not heterogeneous in composition and so often resulted in significant variations when using the dataset inferences directly.

The Demirjian's method of age assessment in 1973, created a scoring criterion based on maturation stages of seven permanent teeth.(6) Although, using this method many studies have shown overestimated chronological age by over a year, many studies still use it for evaluation and comparison with other dental age estimation methods.(1) Few studies tested the accuracy of the Demirjian's method in Saudi population and the results were inconsistent.(7) Willems et al in 2001, simplified Demirjian's technique by creating new tables and maturity score directly expressing age while retaining the benefits of Demirjian's method.(1,6) The Willems method is recommended to estimate chronological age in heterogeneous populations, while Demirjian's method has a broader application as it uses maturity scores.

Al Qahtani et al. in 2010, introduced The London atlas of Human Tooth Development and Eruption which is a comprehensive, evidence-based atlas to overcome some of the limitations of previous atlas and is globally available.(8) This is a simplified atlas based comparative evaluation of the stages of development and is the most recent of the methods that were chosen in our study.

The published methods report a bias when using a single method on small samples of uneven ages. During the early years, methods based on the radiological examinations and evaluations of dental and skeletal development are recommended but in older individuals, accuracies gradually reduce. Current use of age estimation is in matters pertaining to legal responsibility or social rights such as school attendance, social benefits, employment, marriage status, and most importantly for asylum seekers. ${ }^{(9)}$

In order to decrease the bias between different methods for age estimation our study compares the accuracy of dental age estimations by three systems namely the London atlas; Demirjian's method and Willems method. It aims to estimate the age of individuals with three different methods (London Atlas, Demirjian's and Willems methods) in a sample population of Saudi Arabia and to compare relative accuracy of the methods in estimating the chronologic age of an individual. This will enable us to suggest the more suitable method that is applicable in Saudi population.

\section{METHODS}

This retrospective, cross-sectional study was conducted on selected radiographs from 350 subjects of Saudi Arabian population, aged between 4 and 16 years taken from the data base archives of dental clinics of Al Farabi College of Dentistry and two private clinics in Riyadh, Saudi Arabia between January to June 2019.

Clear Orthopantomograms (OPGs) without any position errors or artifacts of subjects who had all teeth of maxillary right, mandibular right \& left quadrant teeth, without any impacted, embedded or trans positioned teeth were included in the study. Radiographs of subjects with any pathology or undergone extraction or restorations of maxillary right, mandibular right \& left quadrant teeth; undergoing orthodontic treatment; congenital/developmental anomalies of jaws and teeth; cross checked on the reception file for any history of craniofacial trauma, jaw lesion/disorder; systemic illness and were excluded from the study. The age group between 4-16 years (48 - 192 months) was selected in this study to include all individuals in each group and to avoid age exclusions in the different groups when using different age estimation methods. For ease of statistical calibration and accuracy, ages were expressed in months.

The same set of radiographs was used in all three methods and the child's chronological age was calculated in months by subtracting the birth date of the subject from the date on which the radiograph was exposed. Then, the dental age estimation was de-identified and analyzed by three trained examiners. All three examiners assessed and scored the stages of teeth development and eruption together. All the scores are then subjected to descriptive statistical analysis.

\section{Demirjian's Method:(10)}

Based on tooth mineralization, the development phases of every one of the seven left permanent mandibular teeth, (from central incisors to second molar), was appraised on 
an 8- stage scale from $\mathrm{A}$ to $\mathrm{H}$, and the criteria for the stages were given independently for every tooth. Each phase of the seven teeth was scored, and the sum of the scores brought about an assessment of the individual's dental 'maturity score' which was estimated on a scale from 0 to 100. The 'maturity score' of every subject was then converted to 'dental age' utilizing standard tables specific for each gender.

\section{Willem's Method:(11)}

The gender specific maturity scores provided by Willems et al was likewise assigned to every tooth independently following the tabulations based on the Demirjian's stages. The total of Willems' scores for every one of the 7 teeth were then tabulated to directly provide a 'dental age' in years dependent on Willem's method.

\section{The London Atlas Method:(12,13)}

A pair of London Atlas illustrations for tooth development and eruption was utilized to compare the relative crown/root length of all the eight right teeth in the upper and lower curves.

\section{Statistical Analysis}

All data analyses were completed using the Excel (version: 365 ProPlus, Microsoft, Redmond, USA). The detailed statistical analysis was done using the IBM SPSS (Statistical Package for Social Science) software (version 19). The significant differences between the chronological age and the estimated dental age was calculated using Paired ' $\mathrm{t}$ ' test ( $\mathrm{P}$ value). The statistical significance was set at $\mathrm{p}<0.05$.

\section{RESULTS}

A total of 350 patients ( 175 males and 175 females) ranging in age from 4 to 16 years were selected and analysed according to gender and age category that included a 12-month range of ages. To assess the significance of the difference between chronological age and the dental age estimated a paired t-test was used. The chronological age was ahead of the estimated age by all three methods used to assess the male samples [Table 1].

\begin{tabular}{|c|c|c|c|c|c|}
\hline & & Mean & $\begin{array}{c}\text { Total } \\
\text { Number }\end{array}$ & S.D. & $\begin{array}{l}\text { Standard } \\
\text { Error } \\
\text { Mean }\end{array}$ \\
\hline \multirow{3}{*}{ Pair 1} & Chronological Age & 114.01 & 175 & 31.113 & 2.352 \\
\hline & Demirjian's Method & 112.58 & 175 & 30.955 & 2.340 \\
\hline & Chronological Age & 114.01 & 175 & 31.113 & 2.352 \\
\hline \multirow[t]{2}{*}{ Pair 2} & William's Method & 110.4320 & 175 & 30.56832 & 2.31075 \\
\hline & Chronological Age & 114.01 & 175 & 31.113 & 2.352 \\
\hline Pair 3 & $\begin{array}{l}\text { The London Atlas } \\
\text { Method }\end{array}$ & 108.61 & 175 & 30.237 & 2.286 \\
\hline \multicolumn{6}{|c|}{$\begin{array}{l}\text { Table 1. Comparison between Chronological Age and Dental Age by } \\
\text { Demirjian's, Willem's and The London Atlas Methods in Males } \\
\text { (Age in Months) Paired t-Test }\end{array}$} \\
\hline
\end{tabular}

The paired t-test showed a significant difference between the chronological age and estimated age using William's Method $(\mathrm{p}=0.000)$ and the London Atlas Method $(\mathrm{p}=0.000)$ and no significant difference between the age estimated by Demirjian's Method ( $\mathrm{p}=0.102)$ and chorological age [Table 2].

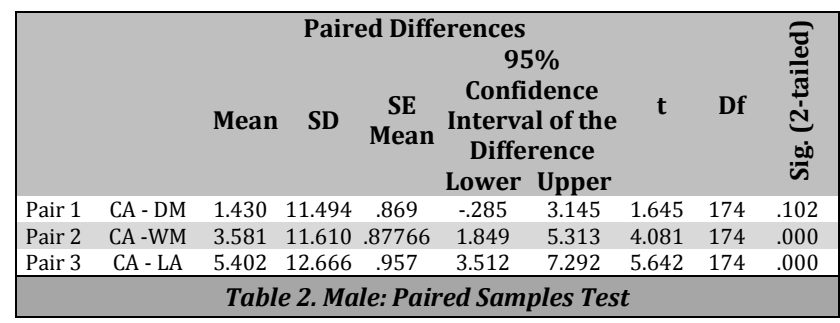

Demirjian's Method estimated the age above chronological age for females while the other two methods were below in their estimates [Table 3].

\begin{tabular}{|cccccc|}
\hline \multirow{4}{*}{ Pair 1 } & Mean & $\begin{array}{c}\text { Total } \\
\text { Number }\end{array}$ & $\begin{array}{c}\text { Standard } \\
\text { Deviation }\end{array}$ & $\begin{array}{c}\text { Standard } \\
\text { Error Mean }\end{array}$ \\
& Chronological Age & 113.89 & 175 & 29.819 & 2.254 \\
& Demirjian's Method & 114.71 & 175 & 29.063 & 2.197 \\
Pair 2 & Chronological Age & 113.89 & 175 & 29.819 & 2.254 \\
& William's Method & 112.8494 & 175 & 29.06855 & 2.19738 \\
& $\begin{array}{c}\text { Chronological Age } \\
\text { Pair } 3\end{array}$ & 113.89 & 175 & 29.819 & 2.254 \\
& $\begin{array}{c}\text { The London Atlas } \\
\text { Method }\end{array}$ & 112.19 & 175 & 30.089 & 2.275 \\
\hline Table 3. Comparison between Chronological Age and Dental Age \\
by Demirjian's, Willem's and The London Atlas Methods in \\
Females (Age in Months) Paired t-Test
\end{tabular}

There were significant differences only between the London atlas estimates and chronological age in females $(p=0.020)$. The other two methods showed no significant differences [Table 4].

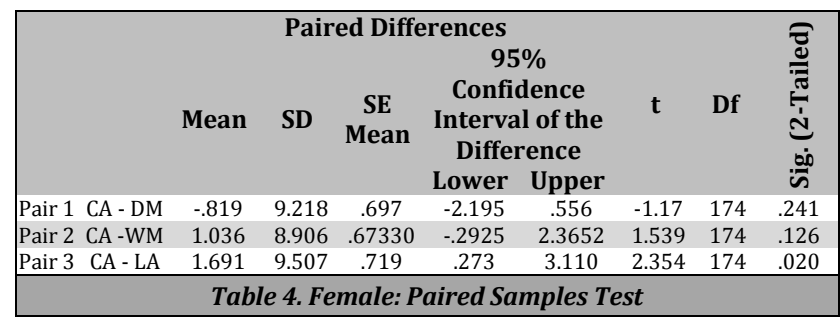

Notes: CA: Chronological Age; DM: Demirjian's Method; WM: William's Method; LA: The London Atlas Method; SD: Standard Deviation; SE mean: Standard Error Mean.

\section{DISCUSSION}

The topic of age determination is of vital importance in many situations in current society and having a reliable method applicable to different populations is key to its applicability. $(3,14)$ The different methods of age estimation using tooth developmental stages have been a main tool used in forensics and law enforcement. $(3,9,10,13)$ These methods have been developed and used for about half a century and they are convenient and reliable for demographic evaluation of age of a population.(14)

The Achilles heel of this approach lies in the fact that the human population is not homogeneous in the patterns of 
growth and development globally. The variations in the phenotypical expressions of genetic variability associated with environmental influences and social changes cause subtle yet confounding changes in the rate of development and chronologic age of different ethnic groups. This problem has been addressed previously and corrections and modifications to the existing methods of age estimation have been proposed time and time again.(14) The variations in growth patterns as a society changes the way it lives and provides for childcare can cause errors in previous estimations that have been suggested for age determination related to a geographical area.(15-17) This necessitates regular updates and re-estimations of age using established methods to make sure that they are applicable as a diagnostic tool, sensitive to be of practical use.(14)

The Saudi population is a prime example of a rapidly changing society that has shown dramatic changes in the socio-economic dynamics over time. These changes could have influenced the factors that affect growth and development. The relative purity in the gene pool and changes over the time in this society focus on the social and environmental impetus as the factors influencing growth.(18) The age estimation of modern-day Saudi population and comparison with earlier studies will show the factual evidence of these influences on growth. In our study we have used three methods of age estimation that have global acceptance. These methods allow for understanding is the relative position of chronologic and growth-related dental age of individuals during the early years of life. The focus of this study was to ascertain the chronologic age of Saudi children using the levels of tooth development as the parameter of assessment. This will allow a verification and recalibration of the corrections needed to make the age determination applicable in contemporary society.

Demirjian's Method was developed to estimate the age of the individual based on the stages observed in teeth.(10) This chart was developed on a French-Canadian population and so has limitations that it needs corrections to be applicable to other ethnic populations. Earlier studies in 2015 on Western Saudi population showed the mean difference of 1.44 to 0.64 in girls and from 0.66 to 0.77 in boys. ${ }^{(8)}$ In our study we found that the Demirjian's Method showed no statistically significant difference from the chronologic age in both males and females. We assume the improved accuracy of the Demirjian's method could be due to the age group range assessed. The Saudi females were behind in chronologic age while the males are seen to be ahead of the estimates as compared to the FrenchCanadian standards of 1973. Changes in diet and habits could have had influences in altering the development of teeth. (15) The reason for this may be due to varying levels of sexual dimorphism or sex-based differences in environmental stresses. (14)

Willem in the year 2001 proposed a new method based on Belgian children who adapted and simplified Demirjian's scoring system and showed increased accuracy of determining chronological ages.(11) In our study there were significant differences in the ages estimated by Willems method for males but not for females. In both cases the estimated ages were less than the chronologic age. The differences in age determined shows a shift in the general development of children and suggests the impact of diet contributes to changes in development of dentition. The growth and development of an individual is dependent on intrinsic and extrinsic factors. The variations in nutritional preferences can significantly alter the growth of individuals.(15-17) Fewer researchers have considered whether the timing of tooth formation varies significantly among human populations. The consistent pattern of variability in overestimation of ages documented by the published studies considered here suggests that variation in the timing of dental development may be influenced by genetic as well as environmental factors. (14)

The London atlas was the most recent of the methods compared in this study as it was developed in 2010.(12,13) There were significant differences between the estimated age and chronologic age in both males and females. In both cases the estimated age was behind the actual age. This indicates that the growth and development of the dataset used in developing the London atlas does vary as compared to the current Saudi population studied and requires further deliberation to discern which factor holds a more profound influence on resulting in differences in dental age.

An interesting finding to consider in the study is that when the Saudi population was assessed in earlier studies there were more deviations in ages determined by the Demirjian's method. $(4,19,20)$ Our study has shown an increased accuracy in this earlier system with the present Saudi population. This could indicate that growth and development of current Saudi children are comparable with the French-Canadian population assessed in mid 70s.

The recent methods developed on European populations showed significant difference in the estimated ages.(20) Here the difference was more evident in the male population showing that the chronological ages preceded the estimated ages. This suggests that the development of the Saudi males could be ahead of the current European patterns. The changes in diet and environment of the urban children studied could influence these findings. A diet abundant in refined carbohydrates and sugary beverages tend to cause increase in the growth and development of the children. The consumption differences that can occur between the males and females will contribute to differences in growth patterns. A study by Dyras et al in 2002 on the Dental age in the relation with nutrition model of school children showed that the differences in the nutrition does influence the development of teeth.(21) This was also noticed in our study as accuracy of the Demirjian's method has increased in comparison to earlier studies done nearly a decade ago.

The study has a few drawbacks that are implied with the limitations of sample size and the association of the differences seen in age estimation with an apparent change in demographic nutritional exposure are only postulates. Further study to quantify changes in diet and effect on dental development is warranted in this population to conclusively decide on its influence.

\section{CONCLUSIONS}

Age estimated by the Demirjian's method in 350 Saudi children was most accurate when compared with their chronologic age. The other methods developed on Caucasian populations estimated ages less than the chronologic age of the children. Boys showed more variation than girls when the ages were measured. Changing diet and socioeconomic factors influence growth and development of children and this can alter the anticipated outcomes of age determination by 
mathematical methods. We also conclude that periodic studies to assess the changes in growth among populations and reassessment of pre-determined correction values for age estimation methods are essential in today's changing society.

We would like to extend our warmest thanks and gratitude to Dr. Sadeq Ali Al-Maweri, Head of the Department of Oral Medicine and Diagnostic Sciences, Al Farabi Colleges, Riyadh, Saudi Arabia, for his expert advice and encouragement throughout this project.

Financial or Other Competing Interests: None.

\section{REFERENCES}

[1] Sathawane RS, Agrawal N. Applicability of ChailletDemirjian's and Willem's age assessment methods in Chhattisgarh population: proposing Chhattisgarh population specific formula. International Journal of Maxillofacial Imaging 2017;3(1):8-11.

[2] AlQahtani S, Kawthar A, AlAraik A, et al. Third molar cutoff value in assessing the legal age of 18 in Saudi population. Forensic Sci Int 2017;272:64-7.

[3] Pavlović S, Pereira CP, de Sousa Santos RFV. Age estimation in Portuguese population: the application of the London atlas of tooth development and eruption. Forensic Sci Int 2017;272:97-103.

[4] Alshihri AM, Kruger E, Tennant M. Dental age assessment of 4-16-year-old Western Saudi children and adolescents using Demirjian's method for forensic dentistry. Egyptian Journal of Forensic Sciences 2016;6:152-6.

[5] Patel PS, Chaudhary AR, Dudhia BB, et al. Accuracy of two dental and one skeletal age estimation methods in 6-16 year-old Gujarati children. J Forensic Dent Sci 2015;7(1):18-27.

[6] Lakshmi Jayaraj MK, Shenoy P. Accuracy of two dental age estimation methods in 6-18 year old children-a radiographic pilot study. IOSR Journal of Dental and Medical Sciences (IOSR-JDMS) 2017;16(9):90-3.

[7] Al-Dharrab AA, Al-Sulaimani FF, Bamashmous MS, et al. Radiographic evaluation of dental age maturity in 3-17years-old saudi children as an indicator of chronological age. J Orthod Sci 2017;6(2):47-53.

[8] Alshihri AM, Kruger E, Tennant M. Dental age assessment of Western Saudi children and adolescents. Saudi Dent J 2015;27(3):131-6.
[9] Alshihri AM, Kruger E, Tennant M. Integrating standard methods of age estimation in western Saudi children and adolescent. Eur J Forensic Sci 2016;3(4):157-62.

[10] Demirjian A, Goldstein H, Tanner JM. A new system of dental age assessment. Hum Biol 1973;45(2):211-27.

[11] Willems G, Van Olmen A, Spiessens B, et al. Dental age estimation in Belgian children: Demirjian's technique revisited. J Forensic Sci 2001;46(4):893-5.

[12] AlQahtani SJ, Hector MP, Liversidge HM. Accuracy of dental age estimation charts: schour and massler, ubelaker and the London atlas. Am J Phys Anthropol 2014;154(1):70-8.

[13] AlQahtani SJ, Hector MP, Liversidge HM. Brief communication: the London atlas of human tooth development and eruption. Am J Phys Anthropol 2010;142(3):481-90.

[14] Esan TA, Yengopal V, Schepartz LA. The Demirjian versus the Willems method for dental age estimation in different populations: a meta-analysis of published studies. PLoS One 2017;12(11):e0186682.

[15] Arid J, Vitiello MC, Silva RAB, et al. Nutricional status is associated with permanent tooth eruption chronology. Brazilian Journal of Oral Sciences 2017;16:1-7.

[16] Vasconcelos KRF, Xavier TA, Oliveira S, et al. Timing of permanent tooth emergence is associated with overweight/obesity in children from the amazon region. Braz Dent J 2018;29(5):465-8.

[17] Dimaisip-Nabuab J, Duijster D, Benzian $H$, et al. Nutritional status, dental caries and tooth eruption in children: a longitudinal study in Cambodia, Indonesia and Lao PDR. BMC Pediatr 2018;18(1):300.

[18] Baghdadi ZD. Dental maturity of Saudi children: role of ethnicity in age determination. Imaging Sci Dent 2013;43(4):267-72.

[19] Alassiry A, Alshomrani K, Al Hasi S, et al. Dental age assessment of 3-15-year-old Saudi children and adolescents using Demirjian's method-a radiographic study. Clin Exp Dent Res 2019;5(4):336-42.

[20] Baghdadi ZD. Dental maturity in Saudi children using the Demirjian method: a comparative study and new prediction models. ISRN Dent 2013;2013:1-9.

[21] Dyras M, Lyszczarz J, Wójtowicz B, et al. Dental age in the relation with nutrition model of school children from swimming classes of championship school. Wiad Lek 2002;55 Suppl 1(Pt 2):662-7. 